\title{
CONTROLE DO Aedes aegypti: AÇÕES EFICIENTES ADOTADAS PELA SECRETARIA DE SAÚDE DE BREJO SANTO EM PARCERIA COM A UNIVERSIDADE FEDERAL DO CARIRI-UFCA
}

\section{CONTROL OF Aedes aegypti: EFFICIENT ACTIONS TAKEN BY THE HEALTH SECRETARY OF BREJO SANTO IN PARTNERSHIP WITH THE FEDERAL UNIVERSITY OF CARIRI-UFCA}

\author{
Edicarlos Pereira dos Santos ${ }^{1}$, Antonio Barros de Souza ${ }^{2}$, Francisca R. Domingos, Gabriel P. Souza, José R. M. \\ Silva
}

Submetido em: $31 / 04 / 2021$

e24193

Aprovado em: 25/05/2021

\section{RESUMO}

Arboviroses são doenças que são acometidas no ser humano através da picada da fêmea do Aedes aegypti infectada, depois do diagnóstico por meio de exame laboratorial podemos saber qual o tipo da doença. Foram realizadas atividades de pesquisa e extensão universitária UFCA e Secretária de Saúde com mutirões educativos nos bairros com maior incidência do vetor, instalação de armadilhas tipo ovitrampas e capacitação de servidores para atuar como brigadistas. As ações do município em parceria com a UFCA conseguiram obter um controle de $71,43 \%$ do índice de infestação do vetor na zona urbana, considerando que o percentual de $28,57 \%$ não é significativo, tendo em vista que o mesmo pode ser relativo à ausência de alguns domicílios por não encontrar moradores, isso gera a possibilidade que esse percentual, considerando este fato, poderia chegar a $90 \%$ ou próximo a $100 \%$ de controle do Aedes aegypti. Este problema epidemiológico é uma ameaça a todos, sendo assim, o estudante universitário assume papel primordial para difundir os conhecimentos adquiridos e gerados na universidade com intuito de contribuir com o desenvolvimento sustentável do município de Brejo Santo-CE.

PALAVRAS-CHAVE: Aedes aegypti. Arboviroses. Controle de vetor. Brejo Santo-CE.

\begin{abstract}
Arboviruses are diseases that are affected in humans through the bite of the infected Aedes aegypti female, after diagnosis through laboratory examination we can know the type of the disease. Research activities and university extension UFCA and Secretary of Health were carried out with educational efforts in the neighborhoods with the highest incidence of the vector, installation of ovitraps-type traps and training of civil servants to act as brigadiers. the actions of the municipality in partnership with UFCA managed to obtain a control of $71.43 \%$ of the vector infestation index in the urban area considering that the percentage of $28.57 \%$ is not significant considering that it may even be related to the absence of some households do not find residents in the households, this generates the possibility that this percentage considering this fact could reach $90 \%$ or close to $100 \%$ control of Aedes aegypti. This epidemiological problem is a threat to everyone, so university students assume a primary role to disseminate the knowledge acquired and generated at the university in order to contribute to the sustainable development of the municipality of Brejo Santo-CE.
\end{abstract}

KEYWORDS: Aedes aegypti, Arboviroses. Vector control. Brejo Santo-CE.

\footnotetext{
${ }^{1}$ Discente do curso de Licenciatura em Química pela (UFCA), Especialização em andamento em Ensino de Química pela Faculdade Única de Ipatinga (FUNIP). É membro do Grupo de Pesquisa e Extensão Educação Ambiental e Controle de endemias na UFCA desde 2018. Possui Licenciatura em Ciências Naturais e Matemática pela Universidade Federal do Cariri - UFCA

2 Mestre em Desenvolvimento Regional Sustentável pela Universidade Federal do Cariri ( 2020), Possui Graduação em Licenciatura Interdisciplinar em Ciências Naturais e Matemática (2017) pela Universidade Federal do Cariri (UFCA). É membro do grupo de pesquisa Agroecologia e desenvolvimento sustentável na Universidade Federal do Cariri - UFCA
} 


\section{RECIMA21 - REVISTA CIENTÍFICA MULTIDISCIPLINAR ISSN 2675-6218}

CONTROLE DO Aedes aegypti: AČ̃ES EFICIENTES ADOTADAS PELA SECRETARIA DE SAÚDE DE BREJO SANTO EM PARCERIA COM A UNIVERSIDADE FEDERAL DO CARIRI-UFCA Edicarlos Pereira dos Santos, Antonio Barros de Souza

\section{INTRODUÇÃO}

Arboviroses são doenças que são acometidas no ser humano através da picada da fêmea do Aedes aegypti infectada, depois do diagnóstico por meio exame laboratorial, podemos saber qual o tipo da doença. São doenças que incluem o vírus da dengue, Zika e Chikungunya, tendo com maior incidência a dengue.

Segundo o Ministério da Saúde, a Dengue pode ser definida como uma doença febril aguda, dinâmica e sistêmica, de notificação compulsória (BRASIL, 2016). A dengue é uma doença típica de áreas tropicais e subtropicais, causada por vírus, transmitida pelo Aedes aegypti (Brasil) e Aedes albopictus (Ásia), geralmente de caráter epidêmico (que atinge um número muito grande de pessoas em um dado momento).

De acordo com a Organização Mundial de Saúde é classificada como vetor a espécie capaz de se infectar com um determinado patógeno via oral, suportar a replicação deste em seu organismo e com isso transmiti-lo a um hospedeiro susceptível. Este processo é chamado de competência vetorial (GUEDES, 2012).

O controle vetorial determinado pela Organização Mundial de Saúde (OMS), inclui a escolha das metodologias mais eficazes a serem empregadas, de acordo com a realidade do local, abrangendo três fases, dentre elas: a definição do local, coleta das informações necessárias e a decisão sobre o momento e a forma de sua implantação (BRAGA; VALLE, 2007).

O agente de combate de endemias (ACE) é fundamental no SUS, atuando nas ações de combate e controle da dengue, realizando inúmeras atividades, dentre elas a visita aos domicílios procurando possíveis focos da doença, alertando e orientando a população, este profissional se encontra na Atenção Básica de Saúde (SANTOS, 2019).

Este estudo tem intuito relatar ações educativas no município de Brejo Santo-CE, a fim de exercer o controle do vetor transmissor da dengue, o Aedes aegypti.

\section{METODOLOGIA}

\section{Delimitação de área de estudo}

A cidade de Brejo Santo, estado do Ceará, faz trabalho de controle ao Aedes Aegypti na sede, incluindo todos os bairros e em algumas localidades da zona rural, onde são deliberados pelo CRES da região, que é a 19a. Conforme dados informados no centro de zoonoses do município, são trabalhados uma quantidade de 20.186 imóveis da sede e da zona rural.

\section{Caracterização das atividades}

Foram realizadas atividades de pesquisa e extensão universitária UFCA e Secretária de Saúde com mutirões educativos nos bairros com maior incidência do vetor, instalação de armadilhas tipo ovitrampas e capacitação de servidores para atuar como brigadistas. 


\section{RECIMA21 - REVISTA CIENTÍFICA MULTIDISCIPLINAR ISSN 2675-6218}

CONTROLE DO Aedes aegypti: ACC̃̃ES EFICIENTES ADOTADAS PELA SECRETARIA DE SAÚDE DE BREJO SANTO EM PARCERIA COM A UNIVERSIDADE FEDERAL DO CARIRI-UFCA Edicarlos Pereira dos Santos, Antonio Barros de Souza

\section{RESULTADOS E DISCUSSÃO}

\section{Ações desenvolvidas para o Controle}

A Policlínica José Gilvan Leite Sampaio em parceria com a Secretaria Municipal de Saúde do Município de Brejo Santo realizou sensibilização com usuários da referida Policlínica sobre "o perigo da dengue persistente". São realizadas regularmente, nesta Policlínica, sensibilizações com o objetivo de alertar a polução dos nove municípios consorciados sobre o perigo de um novo surto da Dengue tipo 2, a qual foi responsável por elevado número de mortes em todo o Estado do Ceará, no ano de 2018. Todos os usuários foram sensibilizados a realizar, semanalmente, vistorias nos mais diversos espaços de suas casas, tais como: caixas d'água, tambores de água, ralos, bandejas de geladeiras, pratos de vasos de plantas, quintais, dentre outros.

Em outro momento, em parceria com a UFCA, ocorreram mutirões educativos nos bairros com maior incidência do vetor, onde profissionais de diversos setores, a prefeitura municipal de Brejo Santo juntamente com alunos da EEP Balbina Viana Arraes e colaboração dos discentes dos Cursos de Licenciatura, Biologia, Química, Ciências Naturais e Matemática, com orientação e apoio dos Professores Coordenadores do Grupo de Extensão e Pesquisa Educação ambiental e Controle de Endemias da UFCA fizeram visitas com as devidas orientações do setor de endemias e também com consentimento da população que eram orientadas sobre a prevenção e controle das arboviroses, além disso, com o consentimento do proprietário, era feita uma vistoria na casa para detectar o vetor Aedes aegypti.

\section{Índice de Infestação do Aedes aegypti}

O trabalho dos agentes de endemias é feito através de ciclos. Exemplo: conforme informação coletada no centro de zoonoses de Brejo Santo, confirmou-se que o município hoje possui 20.186 imóveis atualizados onde é realizado trabalho de combate ao Aedes aegypti.

Durante todos os ciclos de trabalho focal dos agentes de endemias na cidade de Brejo Santo$\mathrm{CE}$, todos tiveram índice de infestação (IIP), ou seja, imóveis positivos para o mosquito. Esses foram dados informados no setor de endemias de Brejo Santo, conhecido também como centro de Zoonoses do Município, esses foram os ciclos de trabalhos dos agentes de endemias durante esses períodos citados, isso significa que o Município tem índice de infestação. Esses dados que foram apresentados servem, para justificar a importância do monitoramento em áreas de risco e de concentração de pessoas.

Os trabalhos para o controle do Aedes aegypti começaram logo no início do ano, pela secretária de Saúde e o departamento de Entomologia, tendo em vista a preocupação dos anos anteriores e a percepção de que o ano 2019 seria um ano difícil, devido ao vírus que circularia e que seria uns dos mais agressivos, tipo 2. 


\section{RECIMA21 - REVISTA CIENTÍFICA MULTIDISCIPLINAR ISSN 2675-6218}

CONTROLE DO Aedes aegypti: AÇÕES EFICIENTES ADOTADAS PELA SECRETARIA BREJO SANTO EM PARCERIA COM A UNIVERSIDADE FEDERAL DO CARIRI-UFCA

Nesse sentido, os trabalhos foram intensificados para não acontecer um surto de Dengue no município. Como mostra a seguinte tabela, as ações foram prioridades para diminuir o foco do vetor.

TABELA 01: Índice de Infestação do Aedes aegypti na zona urbana de Brejo Santo-CE
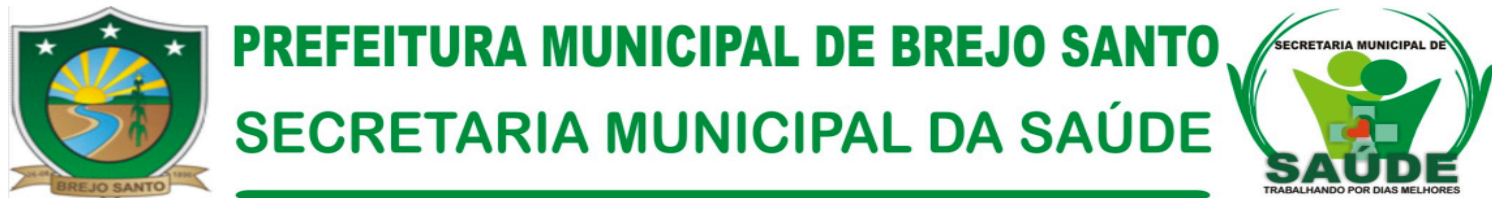

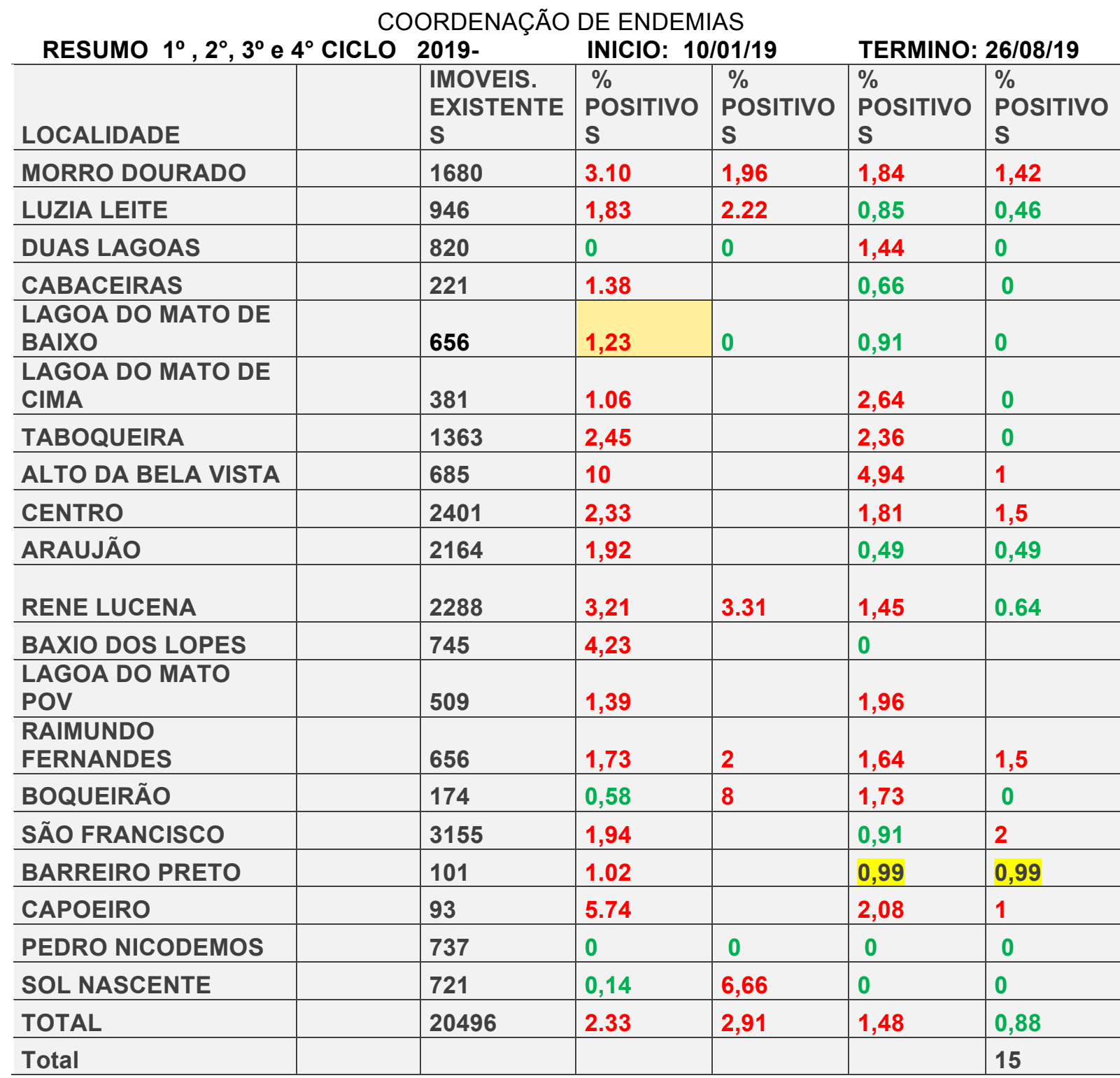

Fonte: Setor de Entomologia/Secretária municipal de Brejo Santo-CE 2019. 

ISSN 2675-6218

Em janeiro de 2019, o qual corresponde ao primeiro ciclo de um período em média 45 dias, aproximadamente $81 \%$ das localidades do município de Brejo Santo-CE , a parte urbana, já sofria com epidemia de infestação, Conforme recomendado pelo ministério da Saúde o índice de infestação deve ser menor que $1 \%$ e nesse ciclo o bairro Alto da Bela Vista estava com índice de $10 \%$ e mais 17 Localidades, também com números alarmantes, causando uma grande preocupação para secretaria de Saúde, a qual não mediu esforços para tentar controlar esse problema no município.

Além disso, o município perdeu os agentes de endemias os quais eram servidores temporários, criando assim mais um desafio para o departamento de Endemias, pelo seu quadro de funcionários reduzido. Nesse sentido, era viável intensificar os trabalhos para suprir essa necessidade do quadro de profissionais.

Como mostra os dados da tabela 01 , fornecido pela secretária de saúde, as ações do município em parceria com a UFCA conseguiram obter um controle de $71,43 \%$ do índice de infestação do vetor na zona urbana, considerando que o percentual de $28,57 \%$ não é significativo, tendo em vista que o mesmo pode ser relativo à ausência de alguns domicílios por não encontrar moradores, fator que gera a possibilidade que esse percentual considerando poderia chegar a $90 \%$ ou próximo a $100 \%$ de controle do Aedes aegypti.

\section{CONSIDERAÇÕES FINAIS}

Com base nos dados apresentados, será de grande importância capacitar pessoas que possam auxiliar no trabalho dos agentes de endemias tendo em vista o número de agentes em exercício. Além disso, este problema epidemiológico é uma ameaça a todos, sendo assim os estudantes universitários assumem papel primordial para difundir os conhecimentos adquiridos e gerados na universidade com intuito contribuir com desenvolvimento sustentável do município de Brejo Santo-CE.

\section{REFERÊNCIAS}

BRAGA, I. A.; VALLE, D. Aedes aegypti: inseticidas, mecanismos de ação e resistência. Epidemiol. Serv. Saúde, Brasília, v. 16, n. 4, p. 279-293, out./dez. 2007.

GUEDES, D. R. D. Análise da competência vetorial para o vírus Dengue em populações naturais de Aedes aegypti e Aedes albopictus de Pernambuco. Recife: Fundação Oswaldo Cruz Centro de Pesquisas Aggeu Magalhães, 2012.

MINISTÉRIO DA SAÚDE. Dengue: Boletim epidemiológico $19^{\circ}$ CRES $.10^{\circ}$ ed. Brasília: Ministério da Saúde, 2019. Disponível em: portalms.saude.gov.br/../2019/.../2019-013-Monitoramento-dos-casosde-arboviroses. Acesso em: 16 maio. 2019. 


\section{RECIMA21 - REVISTA CIENTÍFICA MULTIDISCIPLINAR ISSN 2675-6218}

CONTROLE DO Aedes aegypti: AÇÕES EFICIENTES ADOTADAS PELA SECRETARIA DE SAÚDE DE BREJO SANTO EM PARCERIA COM A UNIVERSIDADE FEDERAL DO CARIRI-UFCA Edicarlos Pereira dos Santos, Antonio Barros de Souza

MINISTÉRIO DA SAÚDE. Dengue: diagnóstico e manejo clínico: adulto e criança. 5. ed. Brasília: Ministério da Saúde, 2016.

SANTOS, L. C. G. B.; SILVA, N. L. Perfil epidemiológico do estado de Alagoas relacionado com à arbovirose dengue. Braz. J. Hea. Rev., Curitiba, v. 2, n. 3, p. 1604-1608, Mar./Apr. 2019. 